\title{
Cerebral vein thrombosis in patients with Philadelphia- negative myeloproliferative neoplasms An European Leukemia Net study
}

Ida Martinelli, ${ }^{1 \star}$ Valerio De Stefano, ${ }^{2}$ Alessandra Carobbio, ${ }^{3}$ Maria L. Randi, ${ }^{4}$ Claudia Santarossa, ${ }^{4}$ Alessandro Rambaldi, ${ }^{5}$ Maria C. Finazzi, ${ }^{5}$ Francisco Cervantes, ${ }^{6}$ Eduardo Arellano-Rodrigo, ${ }^{6}$ Serena Rupoli, ${ }^{7}$ Lucia Canafoglia, ${ }^{7}$ Alessia Tieghi, ${ }^{8}$ Luca Facchini, ${ }^{8}$ Silvia Betti, ${ }^{2}$ Alessandro M. Vannucchi, ${ }^{9}$ Lisa Pieri, ${ }^{9}$ Rossella Cacciola, ${ }^{10}$ Emma Cacciola, ${ }^{10}$ Agostino Cortelezzi, ${ }^{11}$ Alessandra Iurlo, ${ }^{11}$ Enrico M. Pogliani, ${ }^{12}$ Elena M. Elli, ${ }^{12}$ Antonio Spadea, ${ }^{13}$ and Tiziano Barbui ${ }^{3,5}$

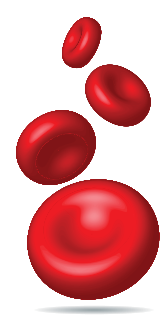

To investigate the characteristics and clinical course of cerebral vein thrombosis (CVT) in patients with myeloproliferative neoplasms (MPN) we compared 48 patients with MPN and CVT (group MPN-CVT) to 87 with MPN and other venous thrombosis (group MPN-VT) and 178 with MPN and no thrombosis (group MPNNoT) matched by sex, age at diagnosis of MPN ( \pm 5 years) and type of MPN. The study population was identified among 5,500 patients with MPN, from January 1982 to June 2013. Thrombophilia abnormalities were significantly more prevalent in the MPN-CVT and MPN-VT than in MPN-NoT group $(P=0.015)$, as well as the JAK2 V617F mutation in patients with essential thrombocythemia $(P=0.059)$. Compared to MPN-VT, MPN-CVT patients had a higher rate of recurrent thrombosis $(42 \%$ vs. $25 \%, P=0.049)$ despite a shorter median follow-up period (6.1 vs. 10.3 years, $P=0.019)$, a higher long-term antithrombotic $(94 \%$ vs. $84 \%, P=$ $0.099)$ and a similar cytoreductive treatment (79\% vs. $70 \%, P=0.311)$. The incidence of recurrent thrombosis was double in MPN-CVT than in MPN-VT group (8.8\% and $4.2 \%$ patient-years, $P=0.022)$, and CVT and unprovoked event were the only predictive variables in a multivariate model including also sex, blood count, thrombophilia, cytoreductive, and antithrombotic treatment (HR 1.97, 95\% $\mathrm{Cl} 1.05-3.72$ and 2.09, 1.09-4.00, respectively).

Am. J. Hematol. 89:E200-E205, 2014. (c) 2014 Wiley Periodicals, Inc.

\section{Introduction}

Essential thrombocythemia, polycythemia vera and myelofibrosis are Philadelphia chromosome-negative chronic myeloproliferative neoplasms (MPN) characterized by a clonal proliferation of an abnormal hematopoietic stem cell [1,2]. MPN can transform into MF and acute leukemia $[3,4]$ and their natural history is distinguished by a tendency towards thrombotic and hemorrhagic complications, that are major causes of patients morbidity and mortality [5-7]. Thrombosis can involve arteries (acute myocardial infarction, ischemic cerebrovascular, or peripheral disease) or veins (deep vein thrombosis at various sites and pulmonary embolism), the former likely more frequent [5-7]. Venous thrombosis (VT) can occur in common sites as the deep circulation of the lower limbs but also in unusual sites. Indeed, typical manifestations of VT in patients with MPN are those of the splanchnic circulation (hepatic, portal, mesenteric, and splenic veins), particular in young women and, to a lesser extent, of the cerebral circulation $[7,8]$. Cerebral vein thrombosis $(\mathrm{CVT})$ is a life-threatening disease that in the general population is rare, accounting for $3-4$ cases every million adults, affects mainly women in fertile age because is strongly associated with the use of oral contraceptives or pregnancy [9]. In patients with MPN the prevalence of CVT is $1 \%$ or less [10-12] and among patients with CVT, MPN is concomitantly diagnosed in 3-7\% of cases [12-14]. In the general population the natural history of CVT is characterized by a relatively good prognosis (80\% of patients who survived the acute phase recover completely) [15] and low recurrence rate of VT, estimated 2-3\% for CVT and 4-7\% for other more common VTs $[13,16]$. No data are available on the natural history of CVT in patients with MPN. This multicenter, observational, retrospective study was aimed to evaluate the prevalence, characteristics, risk factors and clinical course of CVT in a large population of patients with MPN and to compare patients with CVT to those with VT at other sites and those without VT.

${ }^{1}$ Angelo Bianchi Bonomi Hemophilia and Thrombosis, Fondazione IRCCS Ca' Granda-Ospedale Maggiore Policlinico, University of Milan, Italy; ${ }^{2}$ Institute of Hematology, Catholic University, Rome, Italy; ${ }^{3}$ Research Foundation, AO Papa Giovanni XXIII, Bergamo, Italy; ${ }^{4}$ DIMED, Internal Medicine, University of Padua, Italy; ${ }^{5} \mathrm{Hema}-$ tology Division, AO Papa Giovanni XXIII, Bergamo, Italy; ${ }^{6}$ Hospital Clinic, IDIBAPS, University of Barcelona, Spain; ${ }^{7}$ Hematology Clinic, Ospedali Riuniti, Ancona, Italy; ${ }^{8} \mathrm{Hematology}$ Oncology Department, AO Arcispedale Santa Maria Nuova-IRCCS, Reggio Emilia, Italy; ${ }^{9}$ Department of Experimental and Clinical Medicine, University of Florence, Italy; ${ }^{10}$ Haemostasis Unit, Department of Clinical and Molecular Biology, AOU Policlinico Vittorio Emanuele, Catania, Italy; ${ }^{11}$ Hematology and Transplantation Unit, Fondazione IRCCS Ca' Granda-Ospedale Maggiore Policlinico, University of Milan, Italy; ${ }^{12}$ Hematology Division and BMT Unit, San Gerardo Hospital, Milan Bicocca University, Monza, Italy; ${ }^{13}$ Unit of Hematology, Regina Elena National Cancer Institute, Rome, Italy.

Conflicts of interest: Nothing to report

${ }^{\star}$ Correspondence to: Ida Martinelli, Hemophilia and Thrombosis Center, Fondazione IRCCS Ca’ Granda, Ospedale Maggiore Policlinico, Via Pace 9, 20122 Milan, Italy. E-mail: martin@policlinico.mi.it

Contract grant sponsor: Associazione Italiana per la Ricerca sul Cancro -"AIRC 5 per Mille"- to AGIMM, "AIRC-Gruppo Italiano Malattie Mieloproliferative"; Contract grant number: \#1005.

Received for publication: 16 July 2014; Accepted: 16 July 2014

Am. J. Hematol. 89:E200-E205, 2014.

Published online: 19 July 2014 in Wiley Online Library (wileyonlinelibrary.com).

DOI: $10.1002 /$ ajh.23809 
TABLE I. General Characteristics of the Study Population and Comparison of MPN-CVT Patients with MPN-VT or MPN-NoT Patients

\begin{tabular}{|c|c|c|c|c|c|}
\hline & MPN-CVT patients & MPN-VT patients & $p$ & MPN-NoT patients & $p$ \\
\hline$N$ & 48 & 87 & - & 178 & - \\
\hline Median age (range) at diagnosis of MPN & $47(17-84)$ & $46(15-86)$ & 0.871 & $52(18-82)$ & 0.508 \\
\hline Median age (range) at thrombosis & $48(16-83)$ & $52(18-91)$ & 0.764 & - & \\
\hline \multirow{2}{*}{\multicolumn{6}{|c|}{ Type of MPN (n) (\%) }} \\
\hline & & & & & \\
\hline Essential thrombocythemia (ET) & $30(63)$ & $53(61)$ & 0.898 & $105(59)$ & 0.589 \\
\hline Polycythemia vera (PV) & $11(23)$ & $24(28)$ & & $51(28)$ & \\
\hline Primary myelofibrosis (MF) & $6(12)$ & $7(8)$ & & $21(12)$ & \\
\hline Post-ET or post-PV myelofibrosis & $1(2)$ & 3 (3) & & $1(1)$ & \\
\hline JAK2V617F, $n(\%)^{\mathrm{a}}$ & $35(81)$ & $58(83)$ & 0.843 & $110(76)$ & 0.103 \\
\hline JAK2V617F, $n(\%)$ in $\mathrm{ET}^{\mathrm{b}}$ & $19(76)$ & $32(78)$ & 0.847 & $53(55)$ & 0.059 \\
\hline JAK2V617F allelic burden, median (range) & $21(4-91)$ & $31(3-100)$ & 0.455 & $30(2-100)$ & 0.520 \\
\hline MPL mutations, $n(\%)$ & $1(2)$ & 0 & NA & $1(0.5)$ & NA \\
\hline $\mathrm{EEC}, n(\%)$ & $6(13)$ & $8(9)$ & 0.632 & $10(6)$ & 0.406 \\
\hline Abnormal karyotype, $n$ (\%) & $3(6)$ & $3(3)$ & 0.559 & $4(2)$ & 0.092 \\
\hline Thrombophilia screening, $n$ (\%) & $17 / 42(40)$ & 19/55 (35) & 0.549 & $22 / 105(21)$ & 0.015 \\
\hline Antithrombin deficiency & - & - & & 1 & \\
\hline Protein C deficiency & 2 & 4 & & 2 & \\
\hline Protein S deficiency & 2 & 4 & & 6 & \\
\hline Heterozygous factor V Leiden & 2 & 4 & & 2 & \\
\hline Heterozygous prothrombin G20210A & 3 & 1 & & 3 & \\
\hline Hyperhomocysteinemia & 10 & 6 & & 9 & \\
\hline High factor VIII levels & - & - & & - & \\
\hline Antiphospholipid antibodies & 6 & 7 & & 7 & \\
\hline No thrombophilia abnormalities & 25 & 36 & & 83 & \\
\hline Combined thrombophilia abnormalities & 11 & 12 & & 15 & \\
\hline Hematological evolution, $n$ (\%) & $4(8)$ & $10(11)$ & 0.564 & $10(6)$ & 0.331 \\
\hline From PV to MF & - & 1 & & 1 & \\
\hline From ET to MF & 3 & 7 & & 7 & \\
\hline AML/MDS & 1 & 2 & & 2 & \\
\hline Deaths, $n(\%)$ & $4(8)$ & $17(20)$ & 0.135 & $15(8)$ & 0.995 \\
\hline Pulmonary thromboembolism & - & 3 & & - & \\
\hline Budd-Chiari syndrome & 2 & - & & - & \\
\hline Arterial thrombosis & - & 1 & & - & \\
\hline Major bleeding & 1 & - & & 1 & \\
\hline AML/MDS & 1 & 2 & & 2 & \\
\hline Solid tumor & - & 2 & & 1 & \\
\hline Infection & - & - & & 3 & \\
\hline Organ failure & - & 1 & & 2 & \\
\hline Transplant related mortality & - & 1 & & 1 & \\
\hline Other/Unknown & - & 7 & & 5 & \\
\hline
\end{tabular}

MPN myeloproliferative neoplasms; EEC endogenous erythroid colonies; AML acute myeloid leukemia; MDS myelodisplastic syndrome.

a JAK2 status available in 273 patients.

b JAK2 status available in 162 patients.

\section{Methods}

The patient cohort was recruited in 11 Hematology Units, 10 in Italy and one in Spain, in the frame of the European Leukemia Network. Centers were asked to provide information on their regularly followed patients with MPN who developed CVT (group MPN-CVT) with no exclusion criteria but the lack of objective documentation of the thrombotic event. For each MPN-CVT patient, information on 12 patients with MPN who developed objectively documented VT other than CVT (group MPN-VT) and on 3-4 patients with MPN who never had venous or arterial thrombosis (group MPN-NoT) were requested. The index CVT or VT was the first thrombotic event occurred during the follow-up period or within the year preceding the diagnosis of MPN. MPN-VT and MPN-NoT patients should match as much as possible MPN-CVT patients by sex, age at diagnosis of MPN ( \pm 5 years) and type of MPN. Demographic data, location of thrombosis, type of presenting symptoms, results of thrombophilia screening, full blood count at thrombosis, medical history focusing on potential risk factors for thrombosis (infections, trauma, oral contraceptive use, pregnancy, puerperium) and treatment of thrombosis and MPN were recorded in a dedicated data base. In the absence of the aforementioned circumstabtial risk factors for thrombosis the event was considered "unprovoked". Only patients with objectively confirmed diagnosis of CVT (digital angiography, computed tomography angiography, magnetic resonance, or magnetic resonance angiography) or other VT (the same as above but in other body districts, B-mode ultrasound, ventilation/perfusion lung scan) were included. Major bleeding was defined according to the Scientific and Standardization Committee of the International Society on thrombosis and hemostasis [17]. The institutional review boards of the Hospitals approved the study that was carried out and is reported according to the STROBE guidelines for observational studies [18].

Statistical analysis. Summary statistics are presented by groups of interest. For continuous variables, median and 5th-95th percentiles or range are provided. Comparisons were estimated by $t$-test or analogs nonparametric test. For categorical variables, the number and percentage in each category are displayed. Comparison were estimated by Chisquare or Fisher exact test for frequency less than 5 . The relative risk with $95 \%$ confidence interval (CI) was calculated as odds ratio using a $2 \times 2$ contingency table. The annual incidence of recurrent thrombosis was calculated by dividing the number of events by the total number of patient-years. Thrombosis recurrence free-survival was calculated from the date of first thrombotic event to the date of the first recurrence, or to the last followup for censored observations. Kaplan-Meier method was used to estimate survival curves. Cox multivariable model including sex, CVT, blood counts, thrombophilia, unprovoked event, cytoreductive, and antithrombotic treatment was performed in order to find significant predictors of recurrence. Starting with all candidate variables, backward selection was used testing whether the deletion or not of each variable improves the model, and repeating this process until no further improvement is possible.

\section{Results}

\section{Characteristics of MPN patients with thrombosis}

Forty-eight patients formed the MPN-CVT group. Diagnosis of CVT was concomitant to that of MPN in 22 patients (46\%) and was 
TABLE II. Comparison Between MPN-CVT and MPN-VT Patients

\begin{tabular}{|c|c|c|c|}
\hline & MPN-CVT patients & MPN-VT patients & $P$ \\
\hline \multicolumn{4}{|l|}{ Data at CVT/VT } \\
\hline Hemoglobin g/dl, median (range) & $14.0(8.1-20.7)$ & $13.4(8.5-17.6)$ & 0.662 \\
\hline Hematocrit \%, median (range) & $41.5(24.5-62.2)$ & $42.1(26.0-53.3)$ & 0.762 \\
\hline White blood cells $\times 10^{9} / \mathrm{L}$, median (range) & $10.0(2.3-16.6)$ & $8.5(3.7-44.5)$ & 0.179 \\
\hline Platelets $\times 10^{9} / \mathrm{L}$, median (range) & $452(10-1059)$ & $484(7.85-1500)$ & 0.685 \\
\hline Splenomegaly, $n(\%)$ & $11(23)$ & $34(39)$ & 0.076 \\
\hline Risk factors for CVT/VT, $n(\%)$ & $15(31)$ & $23(26)$ & 0.612 \\
\hline Oral contraceptives & 5 & 4 & \\
\hline Hormone replacement therapy & 1 & 1 & \\
\hline Infection & 2 & 2 & \\
\hline Pregnancy/puerperium & 2 & - & \\
\hline Trauma & - & 3 & \\
\hline Solid cancer & - & 5 & \\
\hline Surgery & 6 & 14 & \\
\hline Autoimmune disease & 1 & 2 & \\
\hline Fracture, prolonged immobilization & 1 & 3 & \\
\hline Liver disease & 1 & - & \\
\hline Thrombosis before diagnosis of MPN, $n(\%)^{a}$ & $9(19)$ & $22(25)$ & 0.367 \\
\hline Venous thrombosis & 2 & 10 & \\
\hline Splanchnic vein thrombosis & 3 & 4 & \\
\hline Arterial thrombosis & 4 & 8 & \\
\hline Bleeding before diagnosis of MPN, $n$ (\%) & $1(2)$ & $9(10)$ & 0.073 \\
\hline \multicolumn{4}{|l|}{ Data during follow-up } \\
\hline Median follow-up, years (range) & $6.09(0-34)$ & $10.3(0-31)$ & 0.019 \\
\hline Cytoreductive treatment, $n(\%)$ & $38(79)$ & $61(70)$ & 0.311 \\
\hline Phlebotomy & 6 & 9 & \\
\hline Hydroxyurea & 26 & 49 & \\
\hline Anagrelide & 4 & 4 & \\
\hline Interferon & 6 & 5 & \\
\hline Pipobroman & - & - & \\
\hline Busulphan & - & 2 & \\
\hline Other $^{\mathrm{b}}$ & 2 & 1 & \\
\hline Antithrombotic treatment, $n(\%)$ & $45(94)$ & $73(84)$ & 0.099 \\
\hline Subcutaneous heparin & 4 & 9 & \\
\hline Vitamin $\mathrm{K}$ antagonists & 25 & 39 & \\
\hline Antiplatelet agents & 13 & 19 & \\
\hline Vitamin $\mathrm{K}$ antagonists + antiplatelet agents & 3 & 6 & \\
\hline Cytoreductive + Antithrombotic treatment, $n(\%)$ & $35(73)$ & $57(66)$ & 0.377 \\
\hline Recurrent thrombosis, $n(\%)^{\mathrm{c}}$ & $20(42)$ & $22(25)$ & 0.049 \\
\hline Venous thrombosis & 10 & 11 & \\
\hline Splanchnic vein thrombosis & 6 & 6 & \\
\hline Arterial thrombosis & 4 & 5 & \\
\hline Major bleeding, $n(\%)$ & $7(15)$ & $9(10)$ & 0.412 \\
\hline Central nervous system & 3 & 2 & \\
\hline Gastrointestinal & 1 & 5 & \\
\hline Muscle & 1 & - & \\
\hline Menorrhagia & - & 1 & \\
\hline Epistaxis & 1 & 1 & \\
\hline Hematuria & 1 & - & \\
\hline
\end{tabular}

a Types of thrombosis before index event were 3 superficial, 2 portal, 1 splenic vein thrombosis, 1 acute myocardial infarction, 1 transient ischemic attack, 1 stroke, 1 peripheral artery thrombosis among MPN-CVT patients and 4 superficial, 3 upper limb, 1 lower limb, 1 caval, 1 retinal, vein thrombosis, 3 acute myocardial infarction, 4 transient ischemic attacks, 1 peripheral artery thrombosis among MPN-VT patients.

$\mathrm{b}_{n}=2$ Ruxolitinib, 32P; $n=1$ Vercyte.

c Types of recurrent thrombosis were 9 lower limb deep vein thrombosis and/or pulmonary embolism, 3 portal, 2 hepatic, 1 splenic vein thrombosis, 1 acute myocardial infarction, 2 transient ischemic attacks, 1 peripheral artery thrombosis among MPN-CVT patients and 6 lower limb deep vein thrombosis and/or pulmonary embolism, 4 superficial, 1 upper limb, 3 portal, 1 hepatic, 1 splenic, 1 mesenteric vein thrombosis, 2 transient ischemic attack, 1 stroke, 2 peripheral artery thrombosis among MPN-VT patients.

made within the year before or after diagnosis of MPN in 8 (17\%) and 18 (38\%) patients, respectively, by mean of angio-RM scan in 29 $(60 \%)$, angio-CT scan in $20(42 \%)$, or angiography in $4(8 \%)$. The most frequently involved cerebral sinuses were the superior sagittal in 24 patients (50\%) and the lateral sinus in 21 (44\%). Two or more sinuses were involved in 22 patients $(46 \%)$ and intracranial hemorrhage was present in 11 (23\%). The most common presenting symptom of CVT was headache (85\% of patients), followed by focal neurological defects (50\% of patients had sensory symptoms, visual loss, aphasia, dysarthria, paresis, and vertigo). Only 5 patients (10\%) had impaired consciousness (coma in 2 of them) and 3 (6\%) had seizures. In the acute phase 29 patients $(60 \%)$ were treated with intra- venous or subcutaneous heparin. Forty-five patients (94\%) received long-term antithrombotic treatment that included vitamin $\mathrm{K}$ antagonists in $28(58 \%)$ or antiplatelet agents in $13(27 \%)$ of them. Four patients (9\%) remained in subcutaneous low-molecular weight or unfractionated heparin.

Eighty-seven patients formed the MPN-VT group. Diagnosis of VT was concomitant to that of MPN in 25 patients (29\%). Types of VT were deep vein thrombosis of the lower extremities and/or pulmonary embolism in 66 patients (76\%), of the splanchnic veins in 19 (22\%) and of the upper extremities in $2(2 \%)$. Treatment of acute VT consisted of intravenous or subcutaneous heparin, that was followed by long-term antithrombotic treatment in 73 patients (84\%) and 
TABLE III. Type of Cytoreductive and Antithrombotic Treatment at the Time of Recurrence

\begin{tabular}{|c|c|c|c|}
\hline Index event & Type of recurrent thrombosis & Cytoreductive treatment & Antithrombotic treatment \\
\hline CVT & CVT & Anagrelide & None \\
\hline CVT & DVT + PE & Hydroxyurea & Antiplatelet agents \\
\hline CVT & DVT & None & Subcutaneous heparin \\
\hline CVT & DVT & Hydroxyurea & VKA \\
\hline CVT & DVT & None & VKA \\
\hline CVT & DVT & None & VKA \\
\hline CVT & DVT & None & Subcutaneous heparin \\
\hline CVT & DVT & None & Subcutaneous heparin \\
\hline CVT & $\mathrm{PE}$ & Hydroxyurea & VKA + antiplatelet agents \\
\hline CVT & PE & Hydroxyurea & Not known \\
\hline CVT & Splanchnic VT & Hydroxyurea & Antiplatelet + subcutaneous heparin \\
\hline CVT & Splanchnic VT & Hydroxyurea & VKA + antiplatelet agents \\
\hline CVT & Splanchnic VT & Hydroxyurea & VKA + antiplatelet agents \\
\hline CVT & Splanchnic VT & None & None \\
\hline CVT & Splanchnic VT & None & None \\
\hline CVT & Splanchnic VT & None & Antiplatelet agents \\
\hline CVT & Transient ischemic attack & Hydroxyurea & VKA \\
\hline CVT & Acute myocardial infarction & None & VKA \\
\hline CVT & Peripheral artery thrombosis & None & VKA \\
\hline CVT & Transient ischemic attack & None & VKA \\
\hline OTHER VT & DVT & Interferon & Antiplatelet agents \\
\hline OTHER VT & DVT & None & VKA \\
\hline OTHER VT & DVT & Hydroxyurea & Subcutaneous heparin \\
\hline OTHER VT & DVT & Hydroxyurea & Subcutaneous heparin \\
\hline OTHER VT & $\mathrm{PE}$ & None & Subcutaneous heparin \\
\hline OTHER VT & PE & not known & VKA \\
\hline OTHER VT & PE & Hydroxyurea & Antiplatelet agents \\
\hline OTHER VT & Superficial VT & Hydroxyurea & Antiplatelet agents \\
\hline OTHER VT & Superficial VT & Hydroxyurea & None \\
\hline OTHER VT & Superficial VT & Hydroxyurea & Antiplatelet agents \\
\hline OTHER VT & Superficial VT & Pipobroman & Antiplatelet agents \\
\hline OTHER VT & Splanchnic VT & None & VKA \\
\hline OTHER VT & Splanchnic VT & Hydroxyurea & VKA \\
\hline OTHER VT & Splanchnic VT & None & None \\
\hline OTHER VT & Splanchnic VT & None & Antiplatelet agents \\
\hline OTHER VT & Splanchnic VT & None & None \\
\hline OTHER VT & Splanchnic VT & None & Not known \\
\hline OTHER VT & Ischemic stroke & Interferon & VKA + antiplatelet agents \\
\hline OTHER VT & Transient ischemic attack & Anagrelide & VKA + antiplatelet agents \\
\hline OTHER VT & Peripheral artery thrombosis & None & None \\
\hline OTHER VT & Peripheral artery thrombosis & Hydroxyurea & Antiplatelet agents \\
\hline OTHER VT & Transient ischemic attack & None & Not known \\
\hline
\end{tabular}

CVT, cerebral vein thrombosis; VT, venous thrombosis; DVT, deep vein thrombosis; PE, pulmonary embolism; VKA, vitamin K antagonists.

consisted of vitamin $\mathrm{K}$ antagonists in 45 (52\%) or antiplatelet agents in $19(22 \%)$ of them. Nine patients (10\%) remained in subcutaneous low-molecular weight or unfractionated heparin.

\section{Comparison of patients with and without thrombosis}

Patients were selected from a total population of $\sim 5,500$ patients with MPN followed in the 11 participating Centers until June 2013 (administrative censoring) from the longest period of 31 years (January 1982). Table I shows the characteristics of MPN and the distribution of thrombophilia abnormalities in the study population. Types of MPN were equally distributed in the three groups of patients, being essential thrombocythemia the most represented. At the time of thrombosis MPN-CVT patients were slightly younger than MPN-VT. Median age at recruitment of the MPN-NoT patients was 55 years (range 18-87). Compared to MPN-NoT, MPN-CVT, and MPN-VT patients had a higher prevalence of thrombophilia abnormalities $(21 \%$ vs. $40 \%$ and $35 \% P=0.015)$ and, among those with essential thrombocythemia, of the JAK2 V617F mutation $(76 \%$ and $78 \%$ vs. $55 \%, P=$ 0.059). The development of CVT or VT did not influence the evolution on MPN nor the expectancy of life. Only one death related to VT was recorded in patients who died for pulmonary embolism.

\section{Comparison of MPN-CVT and MPN-VT patients}

Thrombophilia abnormalities (Table I) and other risk factors for thrombosis (Table II) were similarly distributed in MPN-CVT and MPN-VT patients. No differences were seen in the full blood count at the time of thrombosis. MPN-CVT patients had a shorter median follow-up than MPN-VT patients (6.1 vs. 10.3 years, $P=0.019$ ) and received long-term antithrombotic treatment in a higher proportion (94\% vs. $84 \%, P=0.099$ ). The majority of patients in the MPN-CVT and MPN-VT groups received a cytoreductive $(79 \%$ and $70 \%)$ or an anticoagulant treatment ( $94 \%$ and $84 \%)$, mainly hydroxyurea and vitamin K antagonists (Table II). Nine (19\%) MPN-CVT and 22 (25\%) MPN-VT patients had had venous or arterial thrombosis before diagnosis of MPN. Compared to MPN-VT, MPN-CVT patients had a higher rate of recurrent thrombosis (42\% vs. $25 \%, P=$ 0.049 ) that in two-thirds of patients in both groups was venous, with a similar site distribution apart from a slightly higher rate of splanchnic vein thrombosis in MPN-CVT patients (Table II). Also the overall distribution of thrombotic events before or after diagnosis of MPN was similar in the two groups, apart from a slightly higher rate of splanchnic vein thrombosis in MPN-CVT than in MPN-VT patients (19\% vs. $11 \%$, OR 1.77 , 95\%CI $0.66-4.73$ ). 


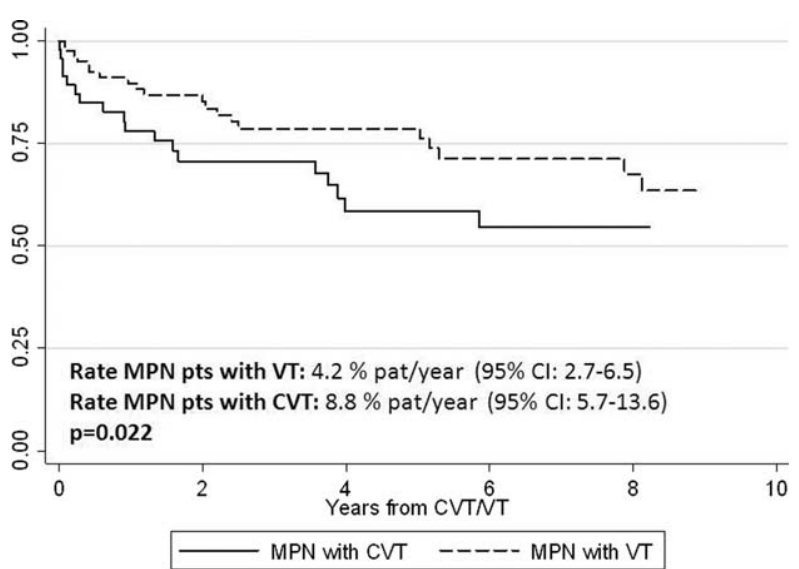

Figure 1. Thrombosis recurrence-free survival in MPN patients with previous CVT or VT.

Details of anticoagulant and cytoreductive treatments at the time of recurrence are shown in Table III. At the time of recurrence, 16 MPN-CVT (80\% of recurrent patients) and 16 MPN-VT patients (73\% of recurrent patients) were on antithrombotic treatment; recurrent thrombosis in patients receiving vitamin $\mathrm{K}$ antagonists (with or without antiplatelet agents), occurred in 10 of 28 (36\%) in the MPNCVT and in 6 of $45(13 \%)$ in the MPN-VT group $(P=0.04)$. On the other hand, recurrent thrombosis in patients receiving antiplatelet agents only occurred in 2 of $13(15 \%)$ in the MPN-CVT and in 7 of $19(37 \%)$ in the MPN-VT group $(P=0.24)$. Finally, recurrent thrombosis in patients receiving hydroxyurea (with or without antithrombotic treatment) occurred in 8 of $26(31 \%)$ in the MPN-CVT and in 8 of $49(16 \%)$ in the MPN-VT group $(P=0.23)$. A combined cytoreductive and antithrombotic treatment was ongoing in 7 of $35 \mathrm{MPN}-$ CVT $(20 \%)$ and in 11 of 57 MPN-VT patients $(19 \%)(P=1.00)$.

CVT recurred in only one patient, who was receiving cytoreductive treatment only. The incidence of recurrent thrombosis was $8.8 \%$ patient-years (95\% CI 5.7-13.6) in MPN-CVT and 4.2\% patient-years (95\% CI 2.7-6.5) in MPN-VT patients (log-rank test, $P=0.022$ ) (Fig. 1). After stepwise selection, a multivariate model including sex, CVT, blood count, thrombophilia, unprovoked event, cytoreductive, and antithrombotic treatments, retained only CVT and unprovoked event as variables predictive of recurrent thrombosis (HR 1.97, 95\%CI $1.05-3.72$ and $2.09,1.09-4.00$, respectively). No difference was seen in the incidence of major bleeding (Table II).

\section{- Discussion}

This study shows that CVT complicated MPN in $\sim 1 \%$ of cases and nearly $50 \%$ of patients have a concomitant diagnosis of the two diseases. The most frequently associated risk factors for CVT, as well as for VT, were thrombophilia abnormalities and, in patients with essential thrombocythemia, the JAK2 V617F mutation. The distribution of thrombophilia abnormalities in patients without thrombosis reflects those observed in the general population, being for the three most common abnormalities factor V Leiden, prothrombin G20210A, and hyperhomocysteinemia $2 \%, 3 \%$, and $10 \%$, respectively. These figures were higher in patients with CVT $(7 \%, 5 \%$, and $24 \%$ ) or VT (7\%, $2 \%$, and $11 \%)$, but much lower than those observed in thrombosis patients without MPN [19]. In the latter, CVT was strongly associated with prothrombin mutation, detected in up to $20 \%$ of cases, approximately as twice as much than factor V Leiden [9]. Our interpretation of this finding is that MPN are per se a strong risk factor for thrombosis and the risk associated with other risk factors appears diluted. Similarly to genetic risk factors, also transient ones such as oral contraceptive or postmenopausal hormone use were less frequent (approximately half the prevalence) either in women with CVT (22\%) or VT $(10 \%)$ than in thrombosis patients without MPN, but the reason of this finding is likely attributable to the relative contraindication to use such hormones in women with an already increased baseline thrombotic risk due to MPN itself. Another interesting finding was a small trend to develop splanchnic vein thrombosis in patients with CVT, but this requires further confirmation.

Treatment of CVT consisted of long-term antithrombotic drugs, mainly vitamin $\mathrm{K}$ antagonists, in almost the totality of patients. Despite of this, MPN-CVT patients had a higher rate of recurrent thrombosis in a shorter follow-up median period of 6 years than MPN-VT patients, with a similar cytoreductive and antithrombotic treatment in the two groups. We noticed that neither cytoreductive therapy with hydroxyurea nor anticoagulant therapy with vitamin $\mathrm{K}$ antagonists was able to prevent recurrent VT in at least one-third of the patients with CVT. Accordingly, at the multivariate analysis neither cytoreductive nor antithrombotic treatment was independently associated with a reduction of recurrent thrombosis.

This finding is partially in contrast with a previous retrospective observation of patients with MPN and previous thrombosis that showed a benefit of either vitamin $\mathrm{K}$ antagonists or antiplatelet agents on the risk of recurrence in patients with a first venous thrombotic event, and a benefit of cytoreductive treatment only in patients with a first arterial thrombosis [20]. Finally, as observed in patients without MPN, also in those with MPN the probability of recurrent CVT is low, occurring in only one patient with essential thrombocythemia and the JAK2 V617F mutation.

Strengths of this study are the relatively large sample of patients with MPN and CVT and the long duration of follow-up that allowed the estimation of the risk of recurrent thrombosis. Among the limitations there is the retrospective design of the study, that impose caution on the interpretation of results on antithrombotic therapy, because some information that are important in the evaluation of the risk of recurrent thrombosis, such as the international normalized ratio of the prothrombin time at the time of recurrence or the median time of patients in therapeutic range are lacking. In addition, the aforementioned interpretation is complicated further by the heterogeneity of antithrombotic treatments, although their distribution was similar in patients with MPN and CVT or VT and there is no reason to think that patients on vitamin $\mathrm{K}$ antagonists for CVT or VT had a different adherence to therapy. Finally, it remains to stratify the patients according to the presence or absence of the recently described calreticulin gene mutations that were not systematically searched in patients without the JAK2 V617F mutation.

In conclusion, among patients MPN those who had a CVT have a higher probability to develop recurrent thrombosis than patients who had VT at other sites than cerebral veins, as well as those who had an unprovoked than a provoked event. Specifically designed studies are needed to assess the optimal prevention of recurrent thrombosis in patients with MPN, in terms of cytoreductive and/or antithrombotic treatments.

\section{Acknowledgments}

For a description of the AGIMM project and list of investigators, see at www.progettoagimm.it.

\section{Author Contributions}

IM designed the study, conceived the data base, wrote the article; VDS designed the study, conceived the data base, included patients and gave a substantial contribution drafting the article; AC performed statistical analyses and critically revised and approved the article; 
MLR, CS, AR, MCF, FC, EAR, SR, LC, AT, LF, SB AMV, LP, RC, EC, AC, AI, EMP, EME, and AS included patients and critically revised and approved the article; TB conceived, designed and coordinated the study, critically revised and approved the article.

\section{References}

1. Tefferi A, Vainchenker W. Myeloproliferative neoplasms: Molecular pathophysiology, essential clinical understanding, and treatment strategies. J Clin Oncol 2011;29:573-582.

2. Tefferi A. Polycythemia vera and essential thrombocythemia: 2013 update on diagnosis, risk-stratification, and management. Am J Hematol 2013;88:507-516.

3. Barbui T, Thiele J, Passamonti F, et al. Survival and disease progression in essential thrombocythemia are significantly influenced by accurate morphologic diagnosis: An international study. J Clin Oncol 2011;29:3179-3184.

4. Tefferi A, Rumi E, Finazzi G, et al. Survival and prognosis among 1545 patients with contemporary polycythemia vera: An international study. Leukemia 2013;27:1874-1881.

5. Patrono C, Rocca B, De Stefano V. Platelet activation and inhibition in polycythemia vera and essential thrombocythemia. Blood 2013;121: 1701-1711.

6. Barbui T, Finazzi G, Falanga A. Myeloproliferative neoplasms and thrombosis. Blood 2013;122: 2176-2184.

7. Casini A, Fontana P, Lecompte TP. Thrombotic complications of myeloproliferative neoplasms: Risk assessment and risk-guided management. J Thromb Haemost 2013;11:1215-1227.

8. Smalberg JH, Arends LR, Valla DC, et al. Myeloproliferative neoplasms in Budd-Chiari syn- drome and portal vein thrombosis: a metaanalysis. Blood 2012;120:4921-4928.

9. Martinelli I, De Stefano V. Rare thromboses of cerebral, splanchnic and upper-extremity veins. A narrative review. Thromb Haemost 2010;103: 1136-1144.

10. Bazzan M, Tamponi G, Schinco P, et al. Thrombosis-free survival and life expectancy in 187 consecutive patients with essential thrombocytemia. Ann Haematol 1999;78:539-543.

11. De Stefano V, Rossi E, Za T, et al. JAK2 V617F mutational frequency in essential thrombocythemia associated with splanchnic or cerebral vein thrombosis. Am J Hematol 2011;86:526-528.

12. Dentali F, Ageno W, Rumi E, et al. Cerebral venous thrombosis and myeloproliferative neoplasms: Results from two large databases. Thromb Res 2014;134:41-43.

13. Ferro JM, Canhão P, Stam J, et al; ISCVT Investigators. Prognosis of cerebral vein and dural sinus thrombosis: Results of the international study on cerebral vein and dural sinus thrombosis (ISCVT). Stroke 2004;35:664-670.

14. Passamonti SM, Biguzzi E, Cazzola $M$, et al. The JAK2 V617F mutation in patients with cerebral venous thrombosis. J Thromb Haemost 2012;10:998-1003.

15. Dentali F, Gianni M, Crowther MA, Ageno W. Natural history of cerebral vein thrombosis: A systematic review. Blood 2006;108:1129-1134.
16. Martinelli I, Bucciarelli P, Passamonti SM, et al. Long-term evaluation of the risk of recurrence after cerebral sinus-venous thrombosis. Circulation 2010;121:2740-2746.

17. Schulman S, Kearon C. Subcommittee on control of anticoagulation of the scientific and standardization committee of the international society on thrombosis and haemostasis. Definition of major bleeding in clinical investigations of antihemostatic medicinal products in non-surgical patients. J Thromb Haemost 2005;3:692-694.

18. Vandenbroucke JP, von Elm E, Altman DG et al. for the STROBE Initiative. Strengthening the Reporting of Observational Studies in Epidemiology (STROBE): Explanation and elaboration. Ann Intern Med 2007;147:W163-W194.

19. Martinelli I, De Stefano V, Mannucci PM. Inherited risk factors for venous thromboembolism. Nat Rev Cardiol 2014;11:140-156.

20. De Stefano V, Za T, Rossi E, et al., GIMEMA CMD-Working Party. Recurrent thrombosis in patients with polycythemia vera and essential thrombocythemia: incidence, risk factors, and effect of treatments. Haematologica 2008;93: 372-380. 